\title{
Effects of laser in situ keratomileusis (LASIK) on corneal biomechanical measurements with the Corvis ST tonometer
}

This article was published in the following Dove Press journal:

Clinical Ophthalmology

12 February 2015

Number of times this article has been viewed

\author{
Andreas Frings ${ }^{1, *}$ \\ Stephan J Linke ${ }^{1,2, *}$ \\ Eva L Bauer' \\ Vasyl Druchkiv' \\ Toam Katz ${ }^{1,2}$ \\ Johannes Steinberg', \\ 'Department of Ophthalmology, \\ University Medical Center Hamburg- \\ Eppendorf (UKE), Hamburg, Germany; \\ ${ }^{2}$ Care Vision Refractive Center, \\ Hamburg, Germany \\ *These authors contributed equally \\ to this work
}

Purpose: This study was initiated to evaluate biomechanical changes using the Corvis ST tonometer (CST) on the cornea after laser in situ keratomileusis (LASIK).

Setting: University Medical Center Hamburg-Eppendorf, Germany, and Care Vision Refractive Centers, Germany.

Design: Retrospective cohort study.

Methods: This retrospective study included 37 eyes of 37 refractive patients. All CST measurements were performed 1 day before surgery and at the 1-month follow-up examination. The LASIK procedure included mechanical flap preparation using a Moria SBK microkeratome and an Allegretto excimer laser platform.

Results: Statistically significant differences were observed for mean first applanation length, mean first and second deflection lengths, mean first and second deflection amplitudes, radius of curvature, and peak distance. Significant positive correlations were found between the change $(\Delta)$ of radius of curvature and manifest refraction spherical equivalent (MRSE), ablation depth, and $\Delta$ intraocular pressure as well as between $\mathrm{AD}$ and $\Delta \mathrm{HC}$-time. Each diopter of myopic correction in MRSE resulted in an increase in $\Delta$ radius of curvature of $0.2 \mathrm{~mm}$.

Conclusion: Several CST parameters were statistically significantly altered by LASIK, thereby indicating that flap creation, ablation, or both, significantly change the ability of the cornea to absorb or dissipate energy.

Keywords: LASIK, corneal biomechanics, refractive surgery, Corvis ST

\section{Introduction}

Refractive surgery alters the biomechanical properties of the cornea, ${ }^{1,2}$ which may play an important role in affecting treatment outcome in terms of postsurgical complications..$^{3,4}$ Roberts $^{5}$ has pointed out that any procedure, such as a laser in situ keratomileusis (LASIK) flap preparation, that circumferentially, or near circumferentially, severs corneal lamellae will produce a biomechanical response that will alter the corneal shape in a manner that cannot easily be predicted. ${ }^{6}$ Thus, in vivo methods for measuring corneal biomechanics could be useful in identifying LASIK candidates and in predicting treatment response.

The Corvis ST tonometer (CST) (Corneal Visualization Scheimpflug Technology, Oculus, Wetzlar, Germany) is a noncontact tonometer (NCT) system integrated with an ultra-high-speed (UHS) Scheimpflug camera that allows investigation of the dynamic reaction of the cornea to an air impulse, thereby quantifying the viscoelastic properties of the cornea and providing intraocular pressure (IOP) information. ${ }^{7}$

The current study was initiated to evaluate biomechanical changes using CST on the cornea after LASIK.
Correspondence: Andreas Frings

Department of Ophthalmology,

University Medical Center Hamburg-

Eppendorf (UKE), Martinistraße 52,

20246 Hamburg, Germany

Tel +49 40741018246

Fax+49407410 54906

Email a.frings@uke.de 


\section{Materials and methods}

\section{Patients and methods}

This retrospective cohort study included eyes from consecutive refractive patients recruited between June and August 2013, and is based on the Hamburg Refractive Database (data retrieved from the Care Vision Refractive Centers, Hamburg, Germany). Informed consent for retrospective data analysis was obtained from refractive surgery candidates during their recruiting process when treatment permission was given, and the study was approved by the local ethics committee (Ethics committee of the University of Hamburg, Hamburg, Germany; No 2882). Our study adhered to the tenets of the Declaration of Helsinki, Finland. Refractive data analyzed herein were assessed preoperatively (preop) and at the 1-month follow-up (Table 1). The spherical and cylindrical refractions were acquired by subjective refraction, and topographic cylinder was obtained using an Orbscan II system (Bausch \& Lomb, Rochester, NY, USA). All patients received CST measurements as standard care within the recruiting process for LASIK surgery and during follow-up appointments.

The principles of the CST have been described in detail elsewhere. ${ }^{7,8}$ Briefly, a frontal view camera was mounted with a keratometer-type projection system for focusing and aligning the corneal apex. We used an automatic release when alignment was achieved with the first Purkinje reflex of the cornea. Preparation of the measurements and patient alignment was done by a trained expert. The UHS Scheimpflug camera takes over 4,000 frames/second to monitor corneal response to a metered, collimated air pulse that forces the cornea inward through applanation into a concavity phase until it achieves the highest concavity (HC). ${ }^{7}$ IOP and central corneal thickness (CCT) are obtained during one measurement process. The biomechanical response of the cornea to the air puff-induced deformation is characterized by devicespecific parameters (see Table 1 and the "Results" section). The applanation length (A length) is the length of a line that describes the applanated part of the cornea (where the cornea is flat). The first A length was measured when the cornea moved downward. The second A length was measured when the cornea passed the applanation phase during the following upward movement.

The deflection length (D length), which describes the deflected part of the cornea compared with the undeformed cornea in the initial state, was also measured. The two end points are fitted to the positions where the shape of the outskirts of the cornea does not differ from the cornea in the initial state. ${ }^{7}$ The deflection amplitude indicates the displacement of the corneal apex with regard to the overlaid cornea in the initial state. The movement of the corneal apex is compensated by the whole eye movement. Only the movement of the cornea is described by this parameter. All CST measurements were performed 1 day before surgery and at the 1-month follow-up examination. A steady head position during the measurement was obtained. Both eyes of each subject were measured; however, eyes with intra- or postoperative complications such as buttonholes or suboptimal postsurgical course, eg, macrostriae, were excluded from the analysis. Further exclusion criteria were ocular pathology or previous ocular surgery despite the current LASIK.

Table I Parameters of 37 eyes of 37 patients before and after LASIK surgery

\begin{tabular}{|c|c|c|c|c|c|c|c|}
\hline \multirow[t]{2}{*}{ Parameters } & \multicolumn{2}{|l|}{ Preop } & \multicolumn{2}{|l|}{ Postop } & \multicolumn{2}{|l|}{ Change } & \multirow[t]{2}{*}{$P^{a}$} \\
\hline & Range & Mean SD & Range & Mean SD & Range & Mean SD & \\
\hline MRSE & -6.00 to 3.63 & $-2.53 \pm 2.38$ & -0.88 to 0.88 & $-0.16 \pm 0.31$ & -6.13 to 3.13 & $-2.37 \pm 2.35$ & 0.000 \\
\hline IOP (mmHg) & $8.50-21.50$ & $14.49 \pm 2.65$ & $7.00-18.00$ & $|2.72 \pm 2.3|$ & -5.00 to 8.00 & $1.77 \pm 2.47$ & 0.000 \\
\hline CCT & $481.00-645.00$ & $563.86 \pm 40.02$ & $395.00-657.00$ & $514.05 \pm 44.64$ & -19.00 to 118.00 & $49.81 \pm 35.74$ & 0.000 \\
\hline Ultrasound CCT & $481.00-656.00$ & $561.19 \pm 38.05$ & $405.00-581.00$ & $505.92 \pm 38.49$ & $20.00-92.00$ & $55.27 \pm 20.64$ & 0.000 \\
\hline First A length (mm) & $|.4|-2.28$ & $1.78 \pm 0.26$ & $1.16-2.19$ & $1.63 \pm 0.26$ & -0.44 to 0.93 & $0.15 \pm 0.32$ & 0.007 \\
\hline Second A length $(\mathrm{mm})$ & I.15-2.56 & $1.90 \pm 0.41$ & $0.87-2.69$ & $1.75 \pm 0.53$ & -1.23 to 1.42 & $0.15 \pm 0.69$ & 0.204 \\
\hline First D length (mm) & $1.93-2.87$ & $2.34 \pm 0.20$ & I.55-2.47 & $2.06 \pm 0.21$ & -0.26 to 0.92 & $0.28 \pm 0.25$ & 0.000 \\
\hline Second $\mathrm{D}$ length $(\mathrm{mm})$ & $1.92-3.25$ & $2.74 \pm 0.29$ & $|.49-3.3|$ & $2.47 \pm 0.40$ & -0.92 to 1.66 & $0.30 \pm 0.52$ & 0.003 \\
\hline First D amplitude (mm) & $0.07-0.13$ & $0.10 \pm 0.02$ & $0.05-0.10$ & $0.08 \pm 0.01$ & -0.02 to 0.06 & $0.02 \pm 0.02$ & 0.000 \\
\hline Second D amplitude (mm) & $0.07-0.17$ & $0.12 \pm 0.03$ & $0.02-0.14$ & $0.09 \pm 0.03$ & -0.05 to 0.11 & $0.02 \pm 0.04$ & 0.001 \\
\hline Radius of curvature (mm) & $5.8 I-9.70$ & $7.79 \pm 0.81$ & $4.94-10.52$ & $7.14 \pm 1.06$ & -1.30 to 2.01 & $0.65 \pm 0.95$ & 0.000 \\
\hline $\mathrm{HC}(\mathrm{ms})$ & $15.94-18.02$ & $16.91 \pm 0.53$ & $15.94-17.33$ & $16.79 \pm 0.37$ & -1.39 to 1.62 & $0.12 \pm 0.64$ & 0.262 \\
\hline Peak distance (mm) & $2.45-5.31$ & $4.77 \pm 0.60$ & $2.44-6.18$ & $4.95 \pm 0.70$ & -3.60 to 2.10 & $-0.17 \pm 0.91$ & $0.021^{1}$ \\
\hline
\end{tabular}

Notes: ${ }^{a}$ Tested with paired $t$-test in cases when the distribution of differences was normal. blf this assumption was violated, Wilcoxon matched-pairs signed-rank test was applied.

Abbreviations: CCT, central corneal thickness; A length, applanation length; D amplitude, deflection amplitude; D length, deflection length; HC, highest concavity; IOP, intraocular pressure; LASIK, laser in situ keratomileusis; MRSE, manifest refractive spherical equivalent; SD, standard deviation. 
All measurements were performed at the same time of the day $(2-4 \mathrm{pm})$ to decrease the effect of diurnal fluctuation. The CST procedure was well tolerated by all the patients included. The CST displays a quality specification grade based on the patients' alignment and the integrity of the data record for every analysis. Only analyses with a high-quality specification grade according to the manufacturer were included in the statistical analyses. The value of the radius describes the radius of curvature at the time of the $\mathrm{HC}$ of the cornea. ${ }^{7}$ The radius of curvature at the time of the $\mathrm{HC}$ was also analyzed according to whether the eyes were myopic or hyperopic. The HC (in milliseconds) reflects the time of the maximum deformation. The highest points of the nondeformed cornea are not necessarily consistent with the end points of the $\mathrm{D}$ length. The peak distance describes the distance between the highest points (peaks) of the nondeformed parts of the cornea.

\section{Surgical procedure}

The LASIK procedure included mechanical flap preparation using an SBK (Moria, Antony, France), with a single-use head with a predefined distance of $90 \mu \mathrm{m}$ between the footplate and the oscillating blade. The SBK is a linear microkeratome (MK) attached to linear tracks on the suction ring, and drives linearly from the temporal cut border to the nasal hinge and back. Both eyes of the same patient were operated on using the same MK and the same head.

Excimer ablation for all eyes was performed using an Allegretto excimer laser platform (Eye-Q $400 \mathrm{~Hz}$, WaveLight $\mathrm{GmbH}$, Erlangen, Germany) under constant eye tracking $(250 \mathrm{~Hz})$. The ablation depth (AD) and the residual bed thickness (RBT) after flap lift (before excimer ablation) were measured by ultrasound pachymetry set on the center of the cornea to rule out systematic differences obtained by CST measurements. The surgical technique and preop, intraoperative, and postop management have been described in detail elsewhere. ${ }^{9}$

\section{Statistical analysis}

Once the data were compiled, they were entered into a spreadsheet program (Microsoft Office Excel; Hamburg Refractive Database) and were statistically analyzed using general purpose statistical software (STATA version 11.0; StataCorp, TX, USA). To compare variables depending on the distribution of differences, a paired $t$-test or a Wilcoxon matched-pairs signed-rank test was applied. To test the hypothesis that the absolute differences were different from zero, a one-sample mean-comparison $t$-test was used.
Depending on the bivariate distributional structure of the data, we applied either ordinary least square or robust regression methods to estimate the functional form of dependence between preop and postop measurements.

Depending on whether the assumption of normal bivariate distribution was satisfied, either Pearson or Spearman correlation coefficients were calculated to evaluate the relationship between the changes ( $\Delta$, delta) of HC time and $\Delta$ radius of curvature versus $\mathrm{AD}, \triangle \mathrm{MRSE}$ (manifest refraction spherical equivalent) (ie, difference between postop and preop MRSE), preop CCT, RBT, and $\triangle \mathrm{IOP}$. A $P$-value less than 0.05 was considered statistically significant. To analyze the reliability of the CST and ultrasound pachymetry measurements, we estimated intraclass correlation.

\section{Results}

All parameters assessed pre- and postop with CST are summarized in Table 1. After exclusion of the eyes that did not meet the above-mentioned inclusion criteria or had CST measurements of a lower quality than that defined by the manufacturer, 37 eyes of 37 patients (mean age $33 \pm 9$ years, range $18-50$ years) were analyzed in this study. The MRSE was $-2.53 \pm 2.38$ diopter (D) (range -6.00 to $3.63 \mathrm{D}$ ) preop and $-0.16 \pm 0.31 \mathrm{D}$ (range -0.88 to $0.88 \mathrm{D})$ postop $(P=0.000$, Table 1).

The mean IOP of all eyes included in the study, obtained with CST, was $14.49 \pm 2.65 \mathrm{mmHg}$ preop and $12.72 \pm 2.31$ $\mathrm{mmHg}$ postop $(P=0.000$, Table 1$)$. Pre- and postop IOP were measured twice, respectively, and the mathematically calculated average data are offered (there were no statistically significant differences between the measurements, respectively). Pre- and postop Corvis-IOP were bivariate normally distributed, and the Pearson's $r$ coefficient was $0.508(P<0.001)$ (Figure 1A and Table 2).

The mean Corvis-measured CCT was 563.86 $440.02 \mu \mathrm{m}$ (range 481-645 $\mu \mathrm{m}$ ) preop and 514.05 $\pm 44.64 \mu \mathrm{m}$ (range $395-657 \mu \mathrm{m})$ postop $(P=0.000)$; both were bivariate normally distributed with a Pearson's $r$ coefficient of $0.649(P=0.000)$ (Figure 1B and Table 2).

To rule out systematic differences in pachymetry data, we compared pachymetry measurements between CST and conventional ultrasound and found no significant differences either pre- or postoperatively between the methods (data not published)

The mean first A length was $1.78 \pm 0.26 \mathrm{~mm}$ (range $1.41-2.28 \mathrm{~mm}$ ) preop and $1.63 \pm 0.26 \mathrm{~mm}$ (range $1.16-2.19 \mathrm{~mm}$ ) postop $(P=0.007)$. Pearson's correlation test was not statistically significant $(P=0.149, r=0.242$, Table 2$)$. The mean 
A

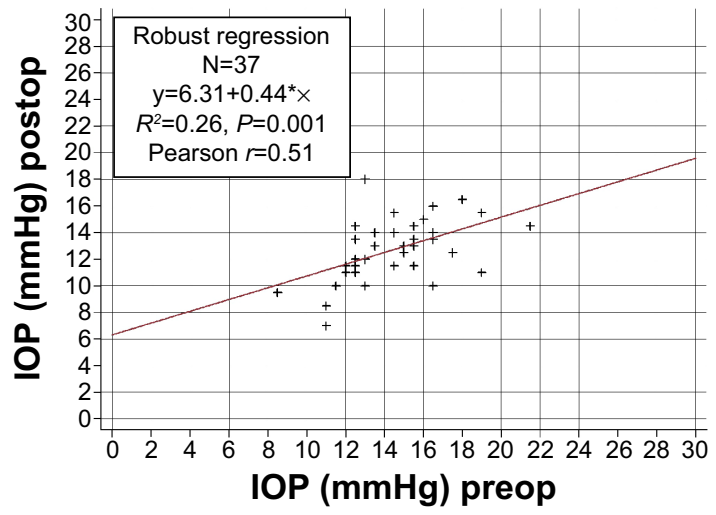

C

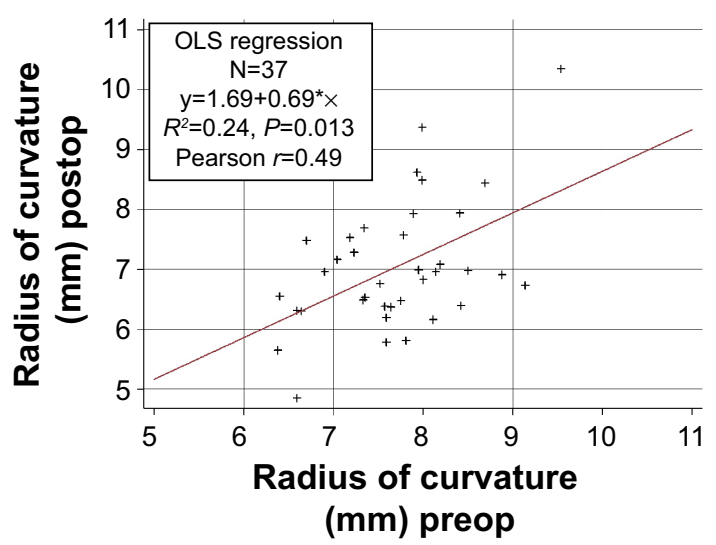

E

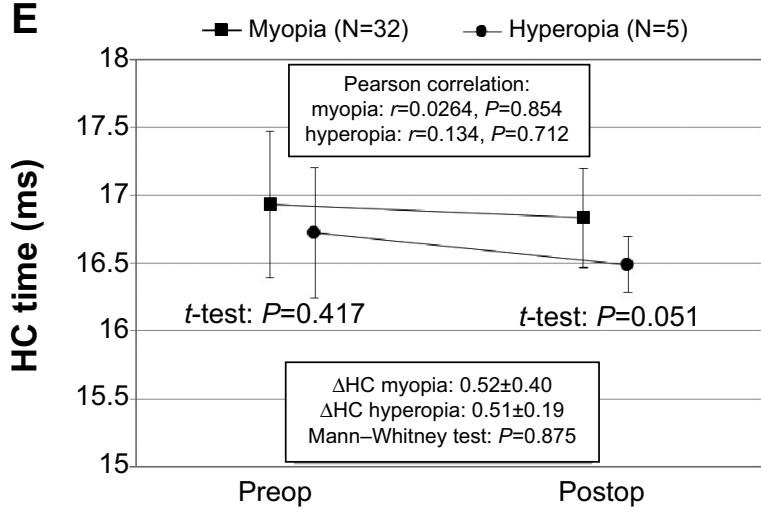

B

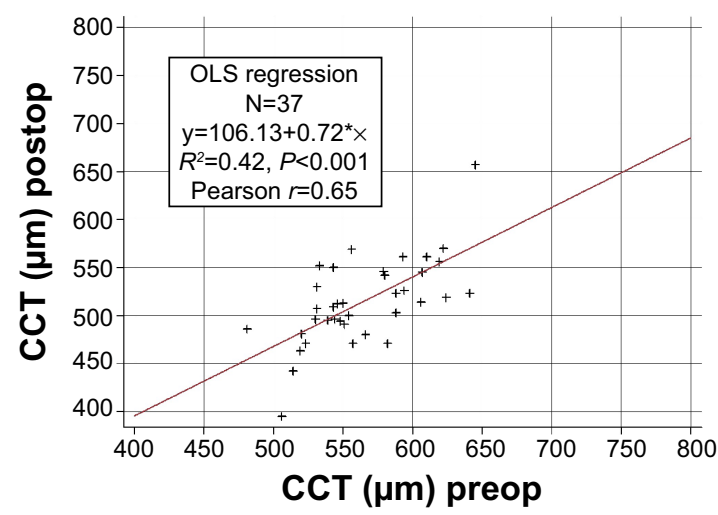

D

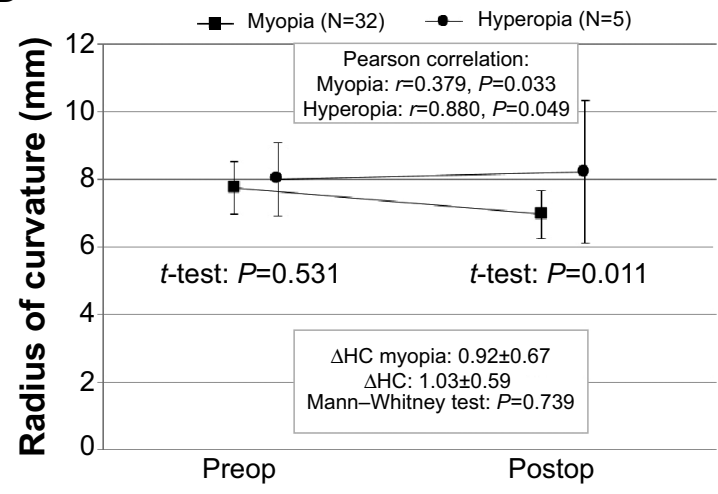

F

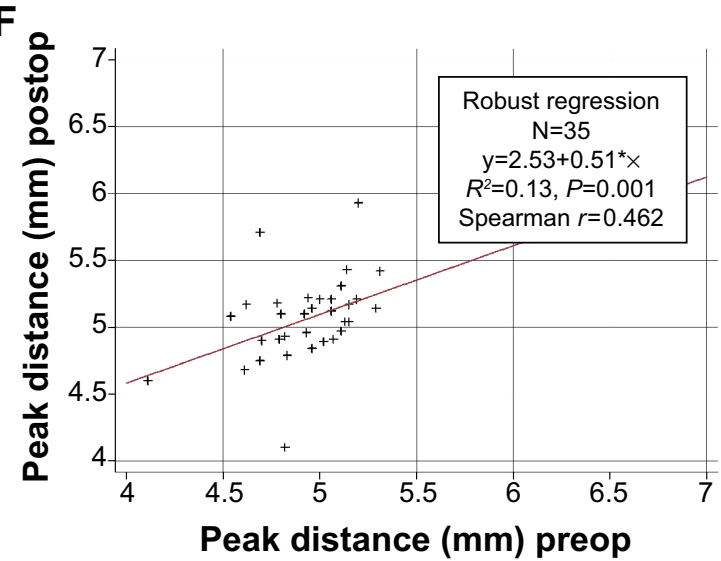

Figure I Selected Corvis ST parameters and their change after LASIK.

Notes: (A) Distribution of IOP preop vs postop measured with CST, (B) distribution of CCT preop vs postop measured with CST, (C) distribution of radius of curvature preop vs postop measured with CST, (D) radius of curvature preop vs postop in hyperopic and myopic eyes, (E) highest concavity (HC in ms) preop vs postop in hyperopic and myopic eyes, and (F) distribution of peak distance preop vs postop measured with CST.

Abbreviations: CCT, central corneal thickness; CST, Corvis ST tonometer; HC, highest concavity; IOP, intraocular pressure; OLS, ordinary least square; preop, before operation; postop, after operation; vs, versus.

second A length was $1.90 \pm 0.41 \mathrm{~mm}$ (range 1.15-2.56 mm) preop and $1.75 \pm 0.53 \mathrm{~mm}$ (range $0.87-2.69 \mathrm{~mm}$ ) postop $(P=0.204)$. Spearman's correlation test was not statistically significant $(P=0.857, r=-0.031$, Table 2$)$.

The mean first D length was $2.34 \pm 0.20 \mathrm{~mm}$ (range $1.93-$ $2.87 \mathrm{~mm}$ ) preop and $2.06 \pm 0.21 \mathrm{~mm}$ (range $1.55-2.47 \mathrm{~mm}$ ) postop $(P=0.000)$. Pearson's correlation test was not statistically significant $(P=0.117, r=0.262$, Table 3$)$. The mean second D length was $2.74 \pm 0.29 \mathrm{~mm}$ (range $1.92-3.25 \mathrm{~mm}$ ) preop and $2.47 \pm 0.40 \mathrm{~mm}$ (range $1.49-3.31 \mathrm{~mm}$ ) postop $(P=<0.003)$. Pearson's correlation test was not statistically significant $(P=0.381, r=-0.162$, Table 2$)$. 
Table 2 Distribution and correlation of data measured with CST

\begin{tabular}{lll}
\hline Parameters & Rho & Sig \\
\hline IOP $(\mathrm{mmHg})$ & 0.508 & 0.00 I \\
CCT $(\mu \mathrm{m})$ & 0.649 & $<0.00$ I \\
First A length $(\mathrm{mm})$ & 0.242 & 0.149 \\
Second A length $(\mathrm{mm})^{\mathrm{a}}$ & $-0.03 \mathrm{I}$ & 0.857 \\
First D length $(\mathrm{mm})$ & 0.262 & $0.1 \mathrm{I}$ \\
Second D length $(\mathrm{mm})$ & -0.162 & $0.38 \mathrm{I}$ \\
First D amplitude $(\mathrm{mm})$ & 0.282 & $0.09 \mathrm{I}$ \\
Second D amplitude $(\mathrm{mm})$ & 0.028 & 0.869 \\
Radius of curvature $(\mathrm{mm})$ & 0.493 & 0.002 \\
HC (ms) & 0.024 & 0.887 \\
Peak distance $(\mathrm{mm})^{\mathrm{a}}$ & 0.462 & 0.004 \\
\hline
\end{tabular}

Notes: ${ }^{\text {a Depending on the underlying joint distribution, either Spearman's or }}$ Pearson's correlation was used (not normally distributed).

Abbreviations: A length, applanation length; CCT, central corneal thickness; CST, Corvis ST tonometer; D amplitude, deflection amplitude; D length, deflection length; HC, highest concavity; IOP, intraocular pressure; Sig, significance.

The mean first deflection amplitude (first $\mathrm{D}$ amplitude) was $0.10 \pm 0.02 \mathrm{~mm}$ (range $0.07-0.13 \mathrm{~mm}$ ) preop and $0.08 \pm 0.01 \mathrm{~mm}$ (range $0.05-0.10 \mathrm{~mm})$ postop $(P=0.000)$. Pearson's correlation test showed borderline insignificance ( $P=0.091, r=0.282$, Table 2 ). The mean second deflection amplitude (second D amplitude) was $0.12 \pm 0.03 \mathrm{~mm}$ (range $0.07-0.17 \mathrm{~mm}$ ) preop and $0.09 \pm 0.03 \mathrm{~mm}$ (range $0.02-0.14 \mathrm{~mm}$ ) postop $(P=0.001)$. Pearson's correlation test was not statistically significant $(P=0.869, r=0.028$, Table 2$)$. These first and second data corresponded to the moment of the first and second applanation events.

The mean radius of curvature preop was $7.79 \pm 0.81 \mathrm{~mm}$ (range 5.81-9.70 $\mathrm{mm}$ ) and postop 7.14 $\pm 1.06 \mathrm{~mm}$ (range 4.94-10.52 mm) $(P=0.000)$ (Figure $1 \mathrm{C})$. Pearson's correlation test was statistically significant $(P=0.002, r=0.493$, Table 2).

The difference was postop statistically significant $(P=0.011)$ as myopic eyes had a statistically significantly

Table 3 Pearson's correlation coefficients ( $\mathrm{N}=37$ eyes)

\begin{tabular}{lll}
\hline Parameters & \multicolumn{2}{r}{ value $(\boldsymbol{P}$-value $)$} \\
\cline { 2 - 3 } & $\Delta$ HC-time & $\Delta$ radius of curvature \\
\hline$\Delta$ MRSE & $0.305^{\text {rs }}$ & $0.4860^{*}$ \\
AD $(\mu \mathrm{m})$ & $0.336^{*, r s}$ & $0.585^{*}$ \\
RBT $(\mu \mathrm{m})$ & $-0.294^{\text {rs }}$ & -0.152 \\
$\Delta \mathrm{IOP}$ & $0.218^{\text {rs }}$ & $0.432^{*, r s}$ \\
Preop CCT & $-0.209^{\text {rs }}$ & 0.26 \\
Treatment zone & $-0.229^{\text {rs }}$ & $-0.059^{\text {rs }}$ \\
size (ablation zone) & & \\
\hline
\end{tabular}

Note: *Significance at 0.05 level.

Abbreviations: $A D$, ablation depth; $C C T$, central corneal thickness; $\mathrm{HC}$, highest concavity; IOP, intraocular pressure; MRSE, manifest refractive spherical equivalent; RBT, residual bed thickness (calculated as ultrasound central pachy "minus" ablation depth "minus" 100); rs, Spearman correlation. smaller radius of curvature measured with CST (Figure 1D).

The mean HC time was $16.91 \pm 0.53 \mathrm{~ms}$ (range 15.94 $18.02 \mathrm{~ms}$ ) preop and $16.79 \pm 0.37 \mathrm{~ms}$ (range $15.94-17.33 \mathrm{~ms}$ ) postop ( $P=0.262$ ). Pearson's correlation test was not statistically significant ( $P=0.887, r=0.024$, Table 2$)$. However, there was borderline insignificance in the $\mathrm{HC}$ time difference when analyzed for myopic and hyperopic eyes $(P=0.051$, Figure 1E).

The mean preop peak distance was $4.77 \pm 0.60 \mathrm{~mm}$ (range $2.45-5.31 \mathrm{~mm}$ ) and $4.95 \pm 0.70 \mathrm{~mm}$ (range 2.44 $6.18 \mathrm{~mm}$ ) postop $(P=0.021)$. Spearman's correlation test was statistically significant $(P=0.004, r=0.462$, Table 2 and Figure 1F).

Significant positive correlations were found between $\Delta$ radius of curvature and $\triangle \mathrm{MRSE}, \mathrm{AD}$ and $\triangle \mathrm{IOP}$, respectively, as well as between $\mathrm{AD}$ and $\Delta \mathrm{HC}$-time (Table 3).

\section{Discussion}

Any procedure that alters corneal biomechanical integrity will have an influence on CST measurement results. ${ }^{5}$ This retrospective study was thus initiated to investigate biomechanical changes using CST on the cornea after LASIK. Consistent with a previous study by Chen et $\mathrm{al}^{10}$ we found that corneal biomechanical capacity was significantly altered after LASIK. Our results demonstrate that flap creation, ablation, or both significantly change the ability of the cornea to absorb or dissipate energy.

Corneal biomechanics involve thickness, hydration, elasticity, viscosity, and other unknown factors including the biomechanical properties of the adjacent scleral tissue. Its behavior is mostly controlled by the stroma, which constitutes $90 \%$ of the total corneal thickness and presents higher mechanical stiffness than the other corneal layers. ${ }^{1,8,11,12}$ Elasticity refers to how a material deforms in response to an external stress. Viscous materials flow when an external shear stress is applied and, unlike materials with elastic properties, do not regain their original shape when the stress is removed. ${ }^{13,14}$ Viscoelasticity refers to a material that displays both viscous and elastic properties, such as the cornea. ${ }^{15}$ Recent studies testing the cohesive tensile strength of the cornea show that the anterior corneal stroma has the greatest cohesive tensile strength ${ }^{16,17}$ and that it is inversely correlated with stromal depth. ${ }^{10}$ During LASIK or any other corneal refractive surgery, we deliberately remove stromal tissue, thereby influencing cohesive tensile strength.

As expected, the CCT was statistically significantly thinner after myopic LASIK. We suggest that changes in 
CST parameters are mainly due to biomechanical changes in the central part of the cornea, because the treatment zone of LASIK is the central cornea. This is supported by statistically significantly shorter A and D lengths as well as a lower deflection amplitude and differences in the radius of curvature in post-LASIK eyes. In addition, the $\mathrm{HC}$ was shorter, whereas the peak distance became longer. This also supports the assumption of a more focused corneal impression in CST measurements due to LASIK. Biomechanically, this could be due to a more asymmetric corneal response to applanation.

The anatomy of the bulbus, the CCT, the IOP itself, the various tractive powers at the surface of the cornea, and particularly its biomechanical properties influence the IOP measurements. ${ }^{18-20}$ The CST is a novel NCT that allows investigation of the dynamic reaction of the cornea to an air impulse. ${ }^{7,21}$ Accurate assessment of corneal thickness is important in many clinical situations, such as in the planning of refractive surgery procedures. We, therefore, investigated the accuracy in pachymetry measurements. The optical corneal thickness measurements from CST showed no significant differences when compared with ultrasound pachymetry. This, in accordance with a previous study on CST, showed excellent repeatability for IOP and pachymetric data. ${ }^{22}$ After myopic LASIK, the central cornea is thinner per diopter of correction. This could cause a false low IOP measurement in cases in which post-LASIK CCT is not addressed. However, the new CST software applied in our analysis compensates for the effect of the measured IOP, as it integrates post-LASIK CCT. Although statistically significantly different, patients presenting with either low or high IOP values showed good agreement between CST measurements in clinical terms (Tables 1 and 2).

Thicker corneas tend to be less deformable and, thus, generate a longer time to applanate and a flatter curvature at $\mathrm{HC}$, which is confirmed by $\mathrm{HC}$, radius of curvature, and applanation time (A1 [not published]). Moreover, myopic eyes had a statistically significantly larger radius of curvature postop. Accordingly, the CST also seems to be not completely unaffected by corneal biomechanical effects. ${ }^{21}$ In addition, preop IOP showed a significantly positive correlation with applanation time A1 (0.4619) and a negative correlation with A length (-0.1022) and velocity $(-0.1879)$ (data on applanation velocity not published). This indicates that a greater IOP causes a higher pressure threshold for the cornea to move, thereby leading to lower deflection.

Applying the latest software update released by the manufacturer in December 2013 enabled us to run our analysis on the basis of selected quality-proven measurements only. However, we acknowledge certain limitations to our study. According to previous studies that compared IOP measurement devices, the CST had the best intraobserver and interobserver variability. ${ }^{21,23,24}$ This is in line with highly repeatable and reproducible results obtained in current healthy corneas (data not published). However, we cannot rule out less repeatability/reproducibility in eyes with prior LASIK, as we measured them only once prior to surgery. Another limitation is the retrospective study design and the relatively small sample size accompanied by a small number of hyperopic eyes. This means that post hoc $P$-values for parameter comparisons should be interpreted with caution because the study was not powered to detect small differences in those parameters. We also did not take age, which could affect the biomechanical properties of the cornea, into account; however, all eyes included here were from young individuals with a mean age of $33 \pm 9$ years (range 18-50).

\section{Conclusion}

The main aim of this study was to evaluate the usefulness of CST measurements in post-LASIK eyes in general, rather than to investigate the influence of various ocular structural properties on different measurements. The recently introduced CST and its wide spectrum of parameters are useful for describing the deformation response of healthy and post-LASIK cornea. Using CST parameters, we found that the corneal biomechanics were statistically significantly altered by LASIK. At present, corneal topography is the most widely used diagnostic tool for screening refractive surgery patients. ${ }^{10,25}$ CST parameters could perhaps also be used as an additional tool in diagnosing healthy versus pathological cornea, in early diagnosis of keratoconus, ${ }^{26}$ and ultimately to establish exclusion criteria for patients at risk for postsurgical ectasia. Moreover, with the help of CST data, preop biomechanical properties of the single cornea could be implemented in surgery planning, to yield more predictable and efficient results after corneal refractive surgery. However, there is still a large gap between "artificial" experimental and daily-routine clinical application of CST measurements in corneal refractive surgery. Further prospective studies are therefore warranted to develop nomograms based on CST measurements.

\section{Acknowledgment}

The abstract of this paper was presented at the XXXII Congress of the European Society of Cataract and Refractive Surgeons (ESCRS) as a poster presentation with interim findings. The poster's abstract was published in "Poster Abstracts" in: http://www.escrs.org/london2014/programme/ posters-details.asp?id=20676. The actual paper, however, has never been published. 


\section{Author contributions}

All authors made substantial contributions to the conception and design, acquisition of data, or analysis and interpretation of data, drafting this article or revising it critically for important intellectual content, gave the final approval of the version to be published, and agree to be accountable for all aspects of the work in ensuring that questions related to the accuracy or integrity of any part of the work are appropriately investigated and resolved.

\section{Disclosure}

None of the authors has any financial or proprietary interest in any materials or methods mentioned, and the authors report no conflicts of interest in this work.

\section{References}

1. Dupps WJ Jr, Wilson SE. Biomechanics and wound healing in the cornea. Exp Eye Res. 2006;83:709-720.

2. Schmack I, Dawson DG, McCarey BE, Waring GO III, Grossniklaus HE, Edelhauser HF. Cohesive tensile strength of human LASIK wounds with histologic, ultrastructural, and clinical correlations. J Refract Surg. 2005;21:433-445.

3. Guirao A. Theoretical elastic response of the cornea to refractive surgery: risk factors for keratectasia. J Refract Surg. 2005;21:176-185.

4. Klein SR, Epstein RJ, Randleman JB, Stulting RD. Corneal ectasia after laser in situ keratomileusis in patients without apparent preoperative risk factors. Cornea. 2006;25:388-403.

5. Roberts C. Biomechanics of the cornea and wavefront-guided laser refractive surgery. J Refract Surg. 2002;18:589-592.

6. Waheed S, Chalita MR, Xu M, Krueger RR. Flap-induced and laserinduced ocular aberrations in a two step LASIK procedure. J Refract Surg. 2005;21:346-352.

7. Oculus Optikgeräte GmbH. Corvis ST Pocket Book. Wetzlar, Germany: Oculus Optikgeräte GmbH. Available from: http://www.oculus.de/en/ sites/detail_ger.php?page1/4597. Accessed June 28, 2012.

8. Valbon BF, Ambrósio R Jr, Fontes BM, Alves MR. Effects of age on corneal deformation by non-contact tonometry integrated with an ultra-high-speed (UHS) Scheimpflug camera. Arq Bras Oftalmol. 2013;76:229-232.

9. Frings A, Katz T, Steinberg J, Druchkiv V, Richard G, Linke SJ. Ocular residual astigmatism: effects of demographic and ocular parameters in myopic laser in situ keratomileusis. J Cataract Refract Surg. 2014;40:232-238.

10. Chen MC, Lee N, Bourla N, Hamilton DR. Corneal biomechanical measurements before and after laser in situ keratomileusis. $J$ Cataract Refract Surg. 2008;34:1886-1891.

11. Boyce BL, Grazier JM, Jones RE, Nguyen TD. Full-field deformation of bovine cornea under constrained inflation conditions. Biomaterials. 2008; 29:3896-3904.

Clinical Ophthalmology

\section{Publish your work in this journal}

Clinical Ophthalmology is an international, peer-reviewed journal covering all subspecialties within ophthalmology. Key topics include: Optometry; Visual science; Pharmacology and drug therapy in eye diseases; Basic Sciences; Primary and Secondary eye care; Patient Safety and Quality of Care Improvements. This journal is indexed on
12. Elsheikh A, Alhasso D, Rama P. Assessment of the epithelium's contribution to corneal biomechanics. Exp Eye Res. 2008;86:445-451.

13. Kotecha A. What biomechanical properties of the cornea are relevant for the clinician? Surv Ophthalmol. 2007;52(Suppl 2):109-114.

14. Hjortdal JO. On the biomechanical properties of the cornea with particular reference to refractive surgery. Acta Ophthalmol Scand Suppl. 1998;(225):1-23.

15. Huseynova T, Waring GO 4th, Roberts C, Krueger RR, Tomita M. Corneal biomechanics as a function of intraocular pressure and pachymetry by dynamic infrared signal and Scheimpflug imaging analysis in normal eyes. Am J Ophthalmol. 2014;157(4):885-893.

16. Randleman JB, Dawson DG, Grossniklaus HE, McCarey BE, Edelhauser HF. Depth-dependent cohesive tensile strength in human donor corneas: implications for refractive surgery. J Refract Surg. 2008;24: S85-S89.

17. Dawson DG, Grossniklaus HE, McCarey BE, Edelhauser HF. Biomechanical and wound healing characteristics of corneas after excimer laser keratorefractive surgery: is there a difference between advanced surface ablation and sub-Bowman's keratomileusis? J Refract Surg. 2008;24:S90-S96.

18. Liu J, Roberts CJ. Influence of corneal biomechanical properties on intraocular pressure measurement: quantitative analysis. J Cataract Refract Surg. 2005;31:146-155.

19. Yu AY, Duan SF, Zhao YE, et al. Correlation between corneal biomechanical properties, applanation tonometry and direct intracameral tonometry. Br J Ophthalmol. 2012;96:640-644.

20. Altan C, Kara N, Baz O, Satana B, Demirok A, Yilmaz OF. Corneal biomechanical properties and intraocular pressure measurement in patients with nanophthalmos. Br J Ophthalmol. 2012;96:806-810.

21. Hong J, Xu J, Wei A, et al. A new tonometer - the Corvis ST tonometer: clinical comparison with noncontact and Goldmann applanation tonometers. Invest Ophthalmol Vis Sci. 2013;54:659-665.

22. Nemeth G, Hassan Z, Csutak A, Szalai E, Berta A, Modis L Jr. Repeatability of ocular biomechanical data measurements with a Scheimpflug-based noncontact device on normal corneas. J Refract Surg. 2013;29:558-563.

23. Moreno-Montañés J, Maldonado MJ, García N, Mendiluce L, GarcíaGómez PJ, Seguí-Gómez M. Reproducibility and clinical relevance of the ocular response analyzer in nonoperated eyes: cornealbiomechanical and tonometric implications. Invest Ophthalmol Vis Sci. 2008;49:968-974.

24. Wang AS, Alencar LM, Weinreb RN, et al. Repeatability and reproducibility of Goldmann applanation, dynamic contour, and ocular response analyzer tonometry. J Glaucoma. 2013;22:127-132.

25. Ambrosio R Jr, Klyce SD, Wilson SE. Corneal topographic and pachymetric screening of keratorefractive patients. J Refract Surg. 2003;19:24-29.

26. Steinberg J, Katz T, Mousli A, et al. Corneal biomechanical changes after crosslinking for progressive keratoconus with the corneal visualization Scheimpflug technology. J Ophthalmol. 2014;2014:579190. doi:10.1155/2014/579190

\section{Dovepress}

PubMed Central and CAS, and is the official journal of The Society of Clinical Ophthalmology (SCO). The manuscript management system is completely online and includes a very quick and fair peer-review system, which is all easy to use. Visit http://www.dovepress.com/ testimonials.php to read real quotes from published authors. 